\title{
Integers: Irreducible Guides in the Search for a Unified Theory
}

\author{
Victor Korotkikh \\ Central Queensland University, Mackay, Queensland, Australia
}

Received on 16 December, 2004

\begin{abstract}
The notion of final theory results from a contrasting understanding of physical reality. Currently, different approaches aim to unify the four forces of nature and discuss whether a final theory may be possible. A key feature of a final theory is irreducibility, however this property has not been seriously exploited. In the paper we present an irreducible mathematical theory that describes physical systems in terms of formation processes of integer relations. The theory has integers and integer relations as the basic elements and is irreducible, because the formation processes are completely controlled by arithmetic. We suggest properties of the formation processes as irreducible guides in the search for a unified theory.
\end{abstract}

\section{Introduction}

The notion of final theory results from a contrasting understanding of physical reality [1]. It relies on beliefs that the most fundamental laws of physics exist and their discovery can be acknowledged. A key feature of a final theory is irreducibility. It must be based on principles that cannot be explained in terms of deeper principles [1]. Currently, different approaches aim to unify the four forces of nature and discuss whether a final theory may be possible [2], [3]. Because the problem is very challenging, technically the irreducibility of a unified theory is not an immediate priority and has not been seriously exploited.

However, a description of physical systems in irreducible terms may reveal a new understanding and opportunities. In particular, the existing approaches will continue to progress from physical observations towards unified theories, but they may be reviewed and improved by guiding through an irreducible theory not necessarily dealing with the forces from the start.

The question arises: where such an irreducible theory may come from? In the paper we address the question and present an irreducible mathematical theory that describes physical systems in terms of formation processes of integer relations. In particular, the theory has the integers and certain integer relations as the basic elements and interprets the relationships between them as a result of their formation processes. The formation processes start with the integers and are completely controlled by arithmetic through a single organizing principle [4]. Consequently, the theory is irreducible as long as the integers are accepted as the most fundamental entities.

We present that the formation processes build up correlation structures of physical systems and determine their properties [5], [6]. As a result the correlations do not take into account the distances between the parts of a physical systems and are nonlocal, instantaneous and inseparable in nature. Furthermore, it turns out that it is possible to geometrize the integer relations as two-dimensional patterns and characterize the formation processes in terms of their geometric transformations [4]. This makes the theory of formation processes geometrical and quantitative.

\section{Correlations Conserving Quantities of Physical Systems}

We approach physical systems from a general perspective. In particular, the dynamics of a physical system is considered in order to understand correlations between the parts. For this purpose we describe a physical system by its certain quantities and are interested to know how many of them remain invariant as the system evolves from one state to another [4].

Let $I$ be an integer alphabet and $I_{N}=\{x=$ $\left.x_{1} \ldots x_{n}, x_{i} \in I, i=1, \ldots, N\right\}$ be the set of all sequences of length $N \geq 2$ with symbols in $I$. Let us consider a physical system consisting of $N$ elementary parts. Let the state of elementary part $i$ be specified by a variable $x_{i} \in I, i=1, \ldots, N$ and the state of the whole physical system by a sequence $x=x_{1} \ldots x_{N}$. Let information about a physical system be given as long as it is known that the system made transition from a state $x=x_{1} \ldots x_{N}$ to another state $x^{\prime}=x_{1}^{\prime} \ldots x_{N}^{\prime}$.

We consider a geometric representation of sequences by using piecewise constant functions. Let $\delta>0$ and $\varepsilon>0$ be length scales of a two-dimensional lattice. Let $\rho_{m \delta \varepsilon}: x \rightarrow g$ be a mapping that realizes the geometric representation of a sequence $x=x_{1} \ldots x_{N} \in I_{N}$ by associating it with a function $g \in W_{\delta \varepsilon}\left[t_{m}, t_{m+N}\right]$, denoted $g=\rho_{m \delta \varepsilon}(x)$, such that the function $g$ is constant on $\left(t_{i-1}, t_{i}\right], i=m+1, \ldots, m+N$, equals $g\left(t_{m}\right)=x_{1} \delta, g(t)=x_{i} \delta, t \in\left(t_{m+i-1}, t_{m+i}\right], i=$ $1, \ldots, N, t_{i}=i \varepsilon, i=m, \ldots, m+N$ and whose integrals satisfy $g^{[k]}\left(t_{m}\right)=0, k=1,2, \ldots$, where $m$ is an integer. The sequence $x=x_{1} \ldots x_{N}$ is called a code of the function $g$ and denoted by $c(g)$.

We use the geometric representation to characterize a state $x=x_{1} \ldots x_{N} \in I_{N}$ of a physical system in terms of 
the definite integrals [4]

$$
V_{\delta \varepsilon}(x, k)=g^{[k]}\left(t_{m+N}\right)=\int_{t_{m}}^{t_{m+N}} g^{[k-1]}(t) d t
$$

of a function $g=\rho_{m \delta \varepsilon}(x) \in W_{\delta \varepsilon}\left[t_{m}, t_{m+N}\right]$, where $g^{[0]}=g$ and $k \geq 1$. The definite integrals use information about the states $x_{i}, i=1, \ldots, N$ of the elementary parts and thus can be viewed as quantities of the physical system in the state $x=x_{1} \ldots x_{N}$.

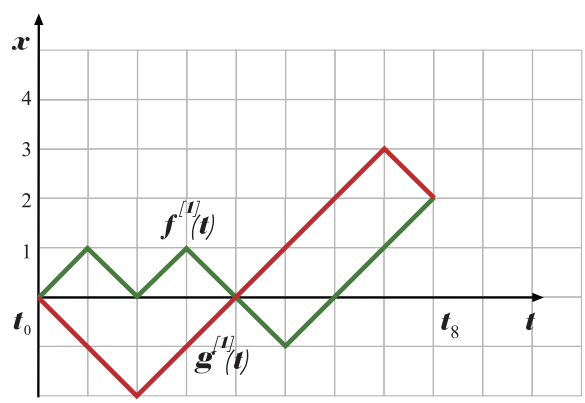

Figure 1. The figure shows that for states $x=-1-1+1+1+$ $1+1+1-1$ and $x^{\prime}=+1-1+1-1-1+1+1+1$ we have $V_{\delta \varepsilon}(x, 1)=V_{\delta \varepsilon}\left(x^{\prime}, 1\right)$, because $g^{[1]}\left(t_{8}\right)=f^{[1]}\left(t_{8}\right)$, where $g=\rho_{m \delta \varepsilon}(x), f=\rho_{m \delta \varepsilon}\left(x^{\prime}\right)$ and $m=0, \delta=1, \varepsilon=1$.

We describe a physical system by the quantities (1) and are concerned with how many of them remain invariant as the system evolves from one state to another. In particular, as a physical system makes transition from a state $x=x_{1} \ldots x_{N}$ to a state $x^{\prime}=x_{1}^{\prime} \ldots x_{N}^{\prime}$ we are interested to know how many of the quantities are conserved [4]

$$
\begin{gathered}
V_{\delta \varepsilon}(x, k)=V_{\delta \varepsilon}\left(x^{\prime}, k\right), \quad k=1, \ldots, C\left(x, x^{\prime}\right), \\
V_{\delta \varepsilon}\left(x, C\left(x, x^{\prime}\right)+1\right) \neq V_{\delta \varepsilon}\left(x^{\prime}, C\left(x, x^{\prime}\right)+1\right),
\end{gathered}
$$

where $V_{\delta \varepsilon}(x, k)=g^{[k]}\left(t_{m+N}\right), \quad V_{\delta \varepsilon}\left(x^{\prime}, k\right)=$ $f^{[k]}\left(t_{m+N}\right), k=1, \ldots, C\left(x, x^{\prime}\right)+1$ and $g=\rho_{m \delta \varepsilon}(x), f=$ $\rho_{m \delta \varepsilon}\left(x^{\prime}\right)$.

The conditions (2) and (3) say that states $x$ and $x^{\prime}$ are the same in terms of the first $C\left(x, x^{\prime}\right) \geq 1$ quantities. But the states $x$ and $x^{\prime}$ are distinct, as they have different values of the $\left(C\left(x, x^{\prime}\right)+1\right)$ th quantity. We consider the conservation of the quantities (2) as a consequence of correlations between the parts of the physical system. To illustrate the notion of the correlations we consider two states of a physical system $x=-1-1+1+1+1+1+1-1$ and $x^{\prime}=+1-1+1-1-1+1+1+1$. In this case $V_{\delta \varepsilon}(x, 1)=$ $V_{\delta \varepsilon}\left(x^{\prime}, 1\right), V_{\delta \varepsilon}(x, 2)=V_{\delta \varepsilon}\left(x^{\prime}, 2\right), V_{\delta \varepsilon}(x, 3) \neq V_{\delta \varepsilon}\left(x^{\prime}, 3\right)$ and $C\left(x, x^{\prime}\right)=2$ (see Figures 1 and 2 ).

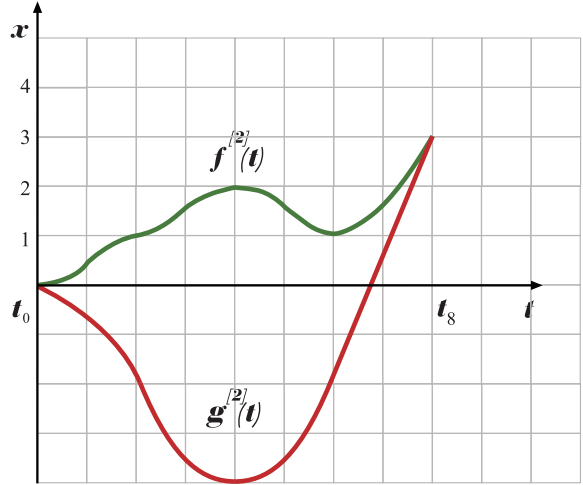

Figure 2. The figure shows that for the states $x$ and $x^{\prime}$ we also have $V_{\delta \varepsilon}(x, 2)=V_{\delta \varepsilon}\left(x^{\prime}, 2\right)$, because $g^{[2]}\left(t_{8}\right)=f^{[2]}\left(t_{8}\right)$.

The sequences $x$ and $x^{\prime}$ do not have obvious regularities and may be even seen as initial segments of random sequences. However, it is interesting to find out how the elementary parts change in order to conserve the two quantities of the physical system as it moves from the state $x$ to the state $x^{\prime}$. The changes can be written as a sequence $x^{\prime \prime}=x_{1}^{\prime \prime} \ldots x_{8}^{\prime \prime}$, where $x_{i}^{\prime \prime}=\Delta x_{i}=x_{i}^{\prime}-x_{i}, i=1, \ldots, 8$. We have $x^{\prime \prime}=+2 \quad 0 \quad 0-2-2 \quad 0 \quad 0+2$. Figure 3 shows the graph of a function $h=\rho_{011}\left(x^{\prime \prime}\right)$. The symmetry of the graph visualizes that the changes of the elementary parts are collectively correlated. It is worth noting that the conservation of the two quantities is connected with the symmetry.

\section{Conserving Correlations as a Sys- tem of Linear Equations}

In section 2 we considered a notion of correlations conserving quantities (1) of a physical system. We are interested to understand the correlations and identify what may connect the parts of a physical system so that a set of the quantities remain invariant.

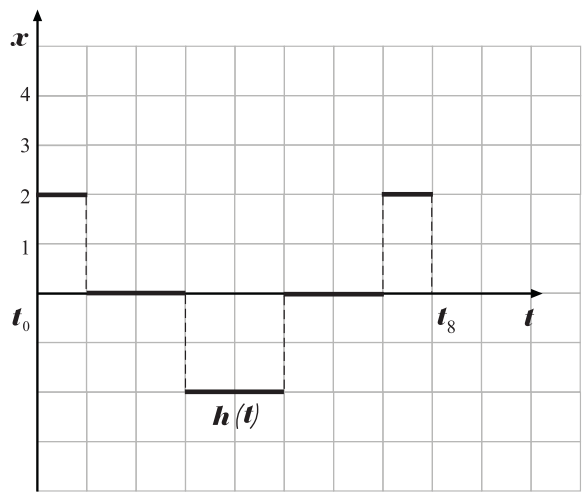

FIgure 3. The symmetry of the graph shows that the changes $\Delta x_{i}, i=1, \ldots, 8$ of the elementary parts are collectively correlated in order to conserve the two quantities of the physical system.

In this section we present that the conditions (2) and (3) can be reduced to a system of linear equations and an inequality [4]. An integer code series [7] plays the key role in the reduction, because it represents an integral of a piecewise constant function $g \in W_{\delta \varepsilon}\left(\left[t_{m}, t_{m+N}\right]\right)$ by using the code $c(g)$ of the function, powers of integers and combinatorial 
coefficients. This expresses the quantities of a physical system in terms of the state explicitly and shows that in order to conserve a set of the quantities the changes of the elementary parts must be correlated as a solution to a system of linear equations [4].

Integer Code Series. Let $g \in W_{\delta \varepsilon}\left(\left[t_{m}, t_{m+N}\right]\right)$ be a piecewise constant function with the code such that $c(g)=$ $x_{1} \ldots x_{N}$. Then the value of the $k$ th $k \geq 1$ integral $g^{[k]}$ of the function $g$ at a point $t_{m+l}, l=1, \ldots, N$ can be written as

$g^{[k]}\left(t_{m+l}\right)=\sum_{i=0}^{k-1} \alpha_{k m i}\left((m+l)^{i} x_{1}+\ldots+(m+1)^{i} x_{l}\right) \varepsilon^{k} \delta$,

where $\alpha_{k m i}, i=1, \ldots, k-1$ are combinatorial coefficients and $m$ is an integer [7].

The integer code series (4) expresses the quantities (2) of a physical system in states $x=x_{1} \ldots x_{N}, x^{\prime}=x_{1}^{\prime} \ldots x_{N}^{\prime}$ as

$$
\begin{gathered}
V_{\delta \varepsilon}(x, k)=g^{[k]}\left(t_{m+N}\right)= \\
\sum_{i=0}^{k-1} \alpha_{k m i}\left((m+N)^{i} x_{1}+\ldots+(m+1)^{i} x_{N}\right) \varepsilon^{k} \delta, \\
V_{\delta \varepsilon}\left(x^{\prime}, k\right)=f^{[k]}\left(t_{m+N}\right)= \\
\sum_{i=0}^{k-1} \alpha_{k m i}\left((m+N)^{i} x_{1}^{\prime}+\ldots+(m+1)^{i} x_{N}^{\prime}\right) \varepsilon^{k} \delta,
\end{gathered}
$$

where $g=\rho_{m \delta \varepsilon}(x), f=\rho_{m \delta \varepsilon}\left(x^{\prime}\right)$ and $k \geq 1$. By using (5) it is proved in [4] that for states $x=x_{1} \ldots x_{N}, x^{\prime}=x_{1}^{\prime} \ldots x_{N}^{\prime} \in$ $I_{N}$ we have

$$
0 \leq C\left(x, x^{\prime}\right) \leq N
$$

and the condition (2) can be written as a system of $C\left(x, x^{\prime}\right) \geq 1$ linear equations

$$
\begin{gathered}
(m+N)^{0} \Delta x_{1}+\ldots+(m+1)^{0} \Delta x_{N}=0 \\
\cdot \quad \cdot \\
(m+N)^{C\left(x, x^{\prime}\right)-1} \Delta x_{1}+\ldots+(m+1)^{C\left(x, x^{\prime}\right)-1} \Delta x_{N}=0
\end{gathered} .
$$

and the condition (3) - as an inequality

$$
(m+N)^{C\left(x, x^{\prime}\right)} \Delta x_{1}+\ldots+(m+1)^{C\left(x, x^{\prime}\right)} \Delta x_{N} \neq 0
$$

in integers $\Delta x_{i}=x_{i}^{\prime}-x_{i}, i=1, \ldots, N$. Moreover, it is demonstrated that $C\left(x, x^{\prime}\right) \geq 1$ of the quantities of a physical system remain invariant iff the changes $\Delta x_{i}=x_{i}^{\prime}-x_{i}, i=$ $1, \ldots, N$ of the elementary parts are correlated as a solution to the system of linear equations (6) and the inequality (7) [4]. The system of linear equations (6) for $m=0$ and $s=0,-1, \ldots,-C\left(x, x^{\prime}\right)+1$ can be written as

$$
\sum_{n=1}^{N} \frac{\Delta x_{N-n+1}}{n^{s}}=0
$$

to show a resemblance with the Dirichlet zeta function

$$
\zeta(s)=\sum_{n=1}^{\infty} \frac{\chi_{n}}{n^{s}}
$$

where $\chi_{n}$ are some coefficients and $s>1$.

\section{The Correlations as Formation Processes of Integer Relations}

In section 3 we presented that correlations defined by conditions (2) and (3) can be characterized by a system of linear equations (6) and an inequality (7). In this section we discuss that the system of linear equations (6) and the inequality (7) can be associated with hierarchical structures of specific integer relations [4]. Furthermore, we interpret the hierarchical structures in terms of formation processes of the integer relations. In such a hierarchical structure the integer relations of one level "form" the integer relations of the higher level [4].

Integer relations that cannot be simply divided into components as integer relations are called the prime integer relations. Their formation processes are especially important. However, it is not known whether the formation processes of prime integer relations may in fact be the formation processes of integer relations.

The system of linear equations (6) links space-time with "reality" of the integer relations. In particular, according to (6) if a physical system changes in space-time, then corresponding formation processes take place in "reality" of the integer relations and as a result produce the correlations between the parts of the physical system.

We illustrate by an example how a hierarchical structure of prime integer relations can be identified from the system of linear equations (6) and the inequality (7), and interpreted in terms of their formation process. Let focus on the coefficients of the system (6) and consider the space-time variables $\Delta x_{i}=x_{i}^{\prime}-x_{i}, i=1, \ldots, N$ as their multipliers. This shows that the coefficients are the consecutive powers $k=0, \ldots, C\left(x, x^{\prime}\right)-1$ of integers $m+N, m+N-1, \ldots, m+1$. From this point of view the system of linear equation (6) is a specific system of integer relations [4]

$$
\Delta x_{1}(m+N)^{0}+\ldots+\Delta x_{N}(m+1)^{0}=0
$$

$$
\Delta x_{1}(m+N)^{C\left(s, s^{\prime}\right)-1}+\ldots+\Delta x_{N}(m+1)^{C\left(x, x^{\prime}\right)-1}=0
$$

and the linear inequality (7) is an inequality between powers of integers

$$
\Delta x_{1}(m+N)^{C\left(x, x^{\prime}\right)}+\ldots+\Delta x_{n}(m+1)^{C\left(x, x^{\prime}\right)} \neq 0 .
$$

For instance, consider two states of a physical system

$x=-1+1+1-1+1-1-1+1+1-1-1+1-1+1+1-1$,

$x^{\prime}=+1-1-1+1-1+1+1-1-1+1+1-1+1-1-1+1$

described by the Prouhet-Thue-Morse (PTM) sequences of length 16 starting with -1 and +1 respectively and $m=$ $0, N=16$. In this case the system of integer relations (8) becomes

$$
+16^{0}-15^{0}-14^{0}+13^{0}-12^{0}+11^{0}+10^{0}-9^{0}
$$




$$
\begin{gathered}
-8^{0}+7^{0}+6^{0}-5^{0}+4^{0}-3^{0}-2^{0}+1^{0}=0, \\
+16^{1}-15^{1}-14^{1}+13^{1}-12^{1}+11^{1}+10^{1}-9^{1} \\
-8^{1}+7^{1}+6^{1}-5^{1}+4^{1}-3^{1}-2^{1}+1^{1}=0, \\
+16^{2}-15^{2}-14^{2}+13^{2}-12^{2}+11^{2}+10^{2}-9^{2} \\
-8^{2}+7^{2}+6^{2}-5^{2}+4^{2}-3^{2}-2^{2}+1^{2}=0, \\
+16^{3}-15^{3}-14^{3}+13^{3}-12^{3}+11^{3}+10^{3}-9^{3} \\
-8^{3}+7^{3}+6^{3}-5^{3}+4^{3}-3^{3}-2^{3}+1^{3}=0
\end{gathered}
$$

and the inequality (9) takes the form

$$
\begin{gathered}
+16^{4}-15^{4}-14^{4}+13^{4}-12^{4}+11^{4}+10^{4}-9^{4} \\
-8^{4}+7^{4}+6^{4}-5^{4}+4^{4}-3^{4}-2^{4}+1^{4} \neq 0,
\end{gathered}
$$

where a common factor 2 originated from the space-time variables $\Delta x_{i}=x_{i}^{\prime}-x_{i}, i=1, \ldots, 16$ is taken of site. We can observe that the correlations are expressed by the signs, which collectively turn the sums in (10) to be 0 . It is worth remarking that the process of calculations in (10) and (11) and its result are completely determined by arithmetic.

An integer relation of (10) may in fact consist of a number of prime integer relations. Prime integers relations may be not directly seen in (10), but can be identified when the powers are selected and tested in a proper way. For example, when the powers are treated from the left to the right, we can find that the first integer relation includes eight prime integer relations

$$
\begin{gathered}
+16^{0}-15^{0}=0,-14^{0}+13^{0}=0, \\
-12^{0}+11^{0}=0,+10^{0}-9^{0}=0,-8^{0}+7^{0}=0, \\
+6^{0}-5^{0}=0,+4^{0}-3^{0}=0,-2^{0}+1^{0}=0 .
\end{gathered}
$$

The second integer relation - four prime integer relations

$$
\begin{aligned}
& +16^{1}-15^{1}-14^{1}+13^{1}=0,-12^{1}+11^{1}+10^{1}-9^{1}=0, \\
& -8^{1}+7^{1}+6^{1}-5^{1}=0, \quad+4^{1}-3^{1}-2^{1}+1^{1}=0 .
\end{aligned}
$$

The third integer relation - two prime integer relations

$$
\begin{gathered}
+16^{2}-15^{2}-14^{2}+13^{2}-12^{2}+11^{2}+10^{2}-9^{2}=0, \\
-8^{2}+7^{2}+6^{2}-5^{2}+4^{2}-3^{2}-2^{2}+1^{2}=0 .
\end{gathered}
$$

The fourth integer relation is a prime integer relation by itself

$$
\begin{gathered}
+16^{3}-15^{3}-14^{3}+13^{3}-12^{3}+11^{3}+10^{3}-9^{3} \\
-8^{3}+7^{3}+6^{3}-5^{3}+4^{3}-3^{3}-2^{3}+1^{3}=0 .
\end{gathered}
$$

The prime integer relations can be defined as the elements of a hierarchical structure. The prime integer relations (12) can be defined as the elements of the first level, the prime integer relations (13) - as the elements of the second level, the prime integer relations (14) - as the elements of the third level and the prime integer relation (15) - as the element of the fourth level.

The reason for this hierarchical organization of the prime integer relations is the existence of certain relationships between them. In the hierarchical structure these relationships become the relationships between the elements of neighboring levels. Furthermore, the hierarchical structure may be interpreted in terms of a formation process of the prime integer relations. Namely, it is considered that the relationships between a prime integer relation of one level and prime integer relations of the lower level exist as a result of the "formation" of the prime integer relation from the prime integer relations.

The formations of the prime integer relations are based on the same organizing principle. For example, the prime integer relation

$$
+16^{2}-15^{2}-14^{2}+13^{2}-12^{2}+11^{2}+10^{2}-9^{2}=0
$$

as an element of the second level can be formed from the prime integer relations

$$
+16^{1}-15^{1}-14^{1}+13^{1}=0,-12^{1}+11^{1}+10^{1}-9^{1}=0
$$

as elements of the first level by using the organizing principle:

- in the left sides of the prime integer relations (17) increase the power of each integer by one

$$
+16^{2}-15^{2}-14^{2}+13^{2},-12^{2}+11^{2}+10^{2}-9^{2},
$$

- combine the results (18) together

$$
+16^{2}-15^{2}-14^{2}+13^{2}-12^{2}+11^{2}+10^{2}-9^{2} .
$$

The sum of (19) equals zero. Thus, we obtain the prime integer relation (16) from the prime integer relations (17) as the result of the above "formation". It is worth noting that the formation of the prime integer relations (17), i.e., the prime integer relation (16), is more than their simple sum.

We have a coherent description of the hierarchical structure and the formation process with integers

$$
16,15,14,13,12,11,10,9,8,7,6,5,4,3,2,1
$$

as elementary building blocks. This requires two additional levels below the four levels of the hierarchical structure (see Figure 4). However, the elements of these two additional levels can be defined in a consistent way with the elements on the other levels. The first additional level may be thought as a "resource" of integers (20) that can "generate" them in "positive" and "negative" states on the second additional level. In the example integers are generated on the level, called the zero level of the structure, in the following states

$$
\begin{gathered}
+16,-15,-14,+13,-12,+11,+10,-9, \\
-8,+7,+6,-5,+4,-3,-2,+1 .
\end{gathered}
$$

The elements of the zero level combine in pairs and form the elements of the first level. Then the formation process proceeds level by level as shown by edges in Figure 4. Notice, that the formation of prime integer relations is more than their simple sum. From Figure 4 we can see that the system of integer relations (10) does not give us much information about the hierarchical structure.

It seems like the formation process has the purpose to progress as higher as possible. As we consider in Figure 4 the process is capable to develop to level 4 , but cannot reach level 5 because arithmetic determines that the left side of (11) cannot be made zero. 


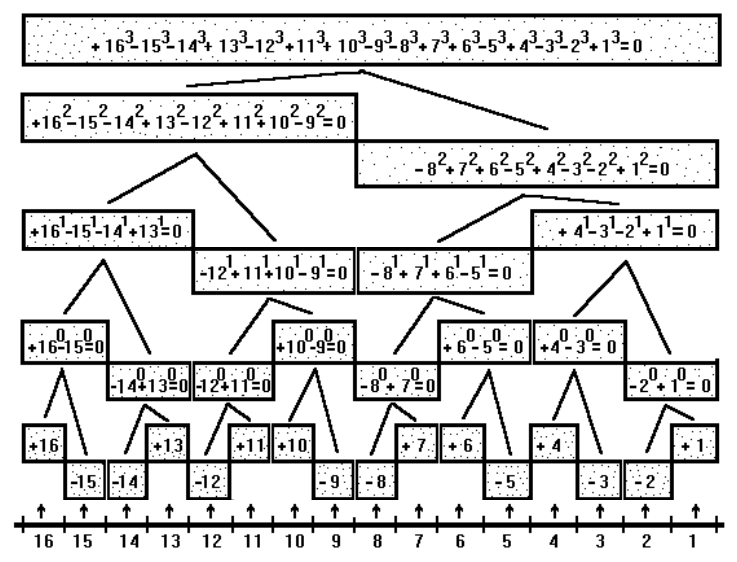

Figure 4. A hierarchical structure of prime integer relations corresponding to the system of integer relations (10) and the inequality (11). The structure may be interpreted in terms of a formation process of the prime integer relations. The formation process starts as integers $16,13,11,10,7,6,4,1$ are "generated" in the "positive" state and integers $15,14,12,9,8,5,3,2$ are "generated" in the "negative" state on the zero level. Then the process goes as arithmetic, through a single organizing principle, manipulates the prime integer relations of one level to form the prime integer relations of the higher level. The formation process cannot progress to level 5, because of (11).

\section{The Formation Processes in the Build up of the Correlation Struc- tures}

In section 4 the system of linear equations (6) and the inequality (7) helped to consider formation processes of integer relations. However, the role of the formation processes for the correlations has not been properly discussed. In this section we present that the formation processes of integer relations build up the correlation structures of physical systems and determine their properties [5], [6]. For example, a formation process of prime integer relations results in a correlation structure, where parts of a physical system are embedded within larger parts.

Formation processes associated with the system of linear equations (6) and the inequality (7) are responsible for the whole structure of the correlations (2) [4]. A part of a physical system may be involved in a number of formation processes and as a result could be included in different elements of the correlation structure. The integer relations play an important role for the correlation structures as they give a representation of the structures and define the correlations.

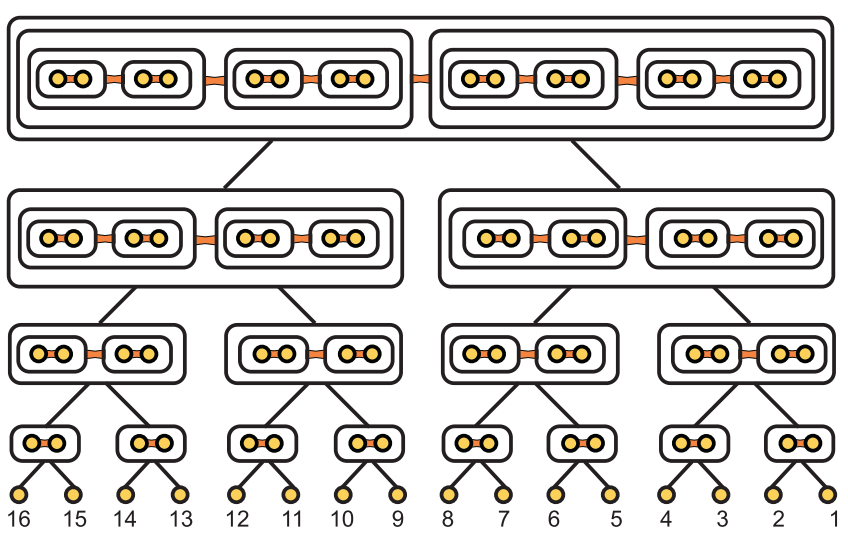

Figure 5. The figure shows how the formation process of prime integer relations described in Figure 4 builds up a correlation structure. The relationship between parts, denoted by an edge, means that the parts are correlated through a prime integer relation. The embedded order of the correlation structure is clearly seen. It is instructive to compare the figure with Figure 4 to see that the form of integer relation is not well suited to give information about the correlation structures.

We illustrate the results by using the formation process described in the previous section. Consider the prime integer relation $+4^{0}-3^{0}=0$. It stands in (8) as

$$
+2 \times 4^{0}-2 \times 3^{0}=\Delta x_{4} 4^{0}+\Delta x_{3} 3^{0}=0
$$

and shows that the elementary parts 4 and 3 have a relationship, because their space-time changes $\Delta x_{4}, \Delta x_{3}$ are correlated

$$
\Delta x_{4} 4^{0}=-\Delta x_{3} 3^{0} .
$$

Therefore, the elementary parts 4 and 3 can be seen as the components of a composite part, denoted by $(4 \leftrightarrow 3)$. The integer relation (21) describes the composite part by telling that it is made of the elementary parts 4 and 3 and they are connected by the correlation (22). Figuratively, the integer relation (21) may seem like a carrier of the "force" of arithmetic that combines the elementary parts 4 and 3 together in the part $(4 \leftrightarrow 3)$.

The integers 4 and 3 play a number of roles in the integer relation (21). They label the dynamics $\Delta x_{4}$ and $\Delta x_{3}$ of the elementary parts 4 and 3 and provide the correlation between them. The correlation through the integer relation (21) does not involve any physical signals. But if the dynamics $\Delta x_{4}$ of the elementary part 4 is specified, then the dynamics $\Delta x_{3}$ of the elementary part 3 is immediately determined as well and vice versa. The composite part $(4 \leftrightarrow 3)$ is schematically represented in Figure 5 by an oval rectangular. It contains two vertices to depict the elementary parts 4 and 3 and an edge between the vertices to indicate the correlation (22). Similarly, the prime integer relation $-2^{0}+1^{0}=0$ describes a composite part $(2 \leftrightarrow 1)$ made of the elementary parts 2 and 1 and the correlation between them

$$
\Delta x_{2} 2^{0}=-\Delta x_{1} 1^{0} .
$$

Let consider the fact that the prime integer relation

$$
+4^{1}-3^{1}-2^{1}+1^{1}=0
$$


is formed from the prime integer relations $+4^{0}-3^{0}=0$ and $-2^{0}+1^{0}=0$. Using the integer relation (23) as it stands in (8)

$$
\left(\Delta x_{4} 4^{1}+\Delta x_{3} 3^{1}\right)+\left(\Delta x_{2} 2^{1}+\Delta x_{1} 1^{1}\right)=0
$$

we can see that the two composite parts $(4 \leftrightarrow 3)$ and $(2 \leftrightarrow 1)$ have a relationship because of the correlation

$$
\left(\Delta x_{4} 4^{1}+\Delta x_{3} 3^{1}\right)=-\left(\Delta x_{2} 2^{1}+\Delta x_{1} 1^{1}\right) .
$$

From the integer relation (25) it follows that the dynamics of the composite part $(4 \leftrightarrow 3)$ is correlated with the dynamics of the composite part $(2 \leftrightarrow 1)$. We may say that the composite parts $(4 \leftrightarrow 3)$ and $(2 \leftrightarrow 1)$ are held together by the "force" of arithmetic exercised through the integer relation (24). Thus, the composite parts $(4 \leftrightarrow 3)$ and $(2 \leftrightarrow 1)$ can be viewed as the components of an even more complex composite part of the physical system, denoted by

$$
((4 \leftrightarrow 3) \leftrightarrow(2 \leftrightarrow 1)) .
$$

The composite part (26) is schematically depicted in Fig. 5 by the right oval rectangular on the second level. The elementary parts $4,3,2$ and 1 are connected by the correlations according to an order of parts within parts. Due to the connections the specification of the dynamics of any elementary part immediately determines the dynamics of the other parts.

In general, the formation process starts with the elementary parts $16,15, \ldots, 1$ and combine them in pairs to make composite parts, which in their turn also combine in pairs to make even more complex composite parts and so on until the correlation structure is built (see Fig. 5). The relationship between parts, denoted by an edge, means that the changes of the parts are correlated through a prime integer relation.

To characterize the correlation structure in Figure 5 we may compare it with a "particle", which is made of two correlated "particles", which in their turn are also made of two correlated "particles" and so on, until the elementary parts are eventually found. As we view in Figure 4 all the "particles" are connected by the "force" of arithmetic.

\section{The Formation Processes as an Irre- ducible Theory}

In sections 4 and 5 we described the formation processes of integer relations. An important distinction of these processes is that they are irreducible. This property comes because the formation processes are based on the integers and completely controlled by arithmetic. In particular, integer relations can form another integer relations only if arithmetic equates corresponding expressions to zero and they cannot do that if it does not.

The formation processes organize the integer relations into a hierarchical network structure, where the integer relations seem interconnected, interdependent and inseparable elements. Each integer relation is a statement, whose truth is completely determined by arithmetic. Thus, the irreducibility of the formation processes is based on the fact that the integer relations, for example such as $3^{2}+4^{2}-5^{2}=0$ and $2+4-5 \neq 0$, are ultimate truths and cannot be further explained.

The formation processes are suggested as a conceptual tool in order to interpret the relationships between the integer relations. Yet in this form the integer relations behave in such a way that it becomes more convenient to think about them as "particles". The term "particle" helps to see the integers and the integer relations not as objects of calculation, but as objects of formation with specific properties:

- The integer relations are formed from the integers. The integer relations of the first level are formed as prime integer relations. From the reverse perspective, an integer relation can be broken down into integer relations of the lower level, where the integer relations in their turn can be further broken down unless they are integers.

- Integer relations combine and form integer relations according to a single organizing principle. An integer relation is more than the simple sum of the integer relations it is formed of.

- An integer relation may participate in various formation processes and as a result be involved in different integer relations of higher levels.

- The integer relations do not stand alone by themselves, but due to the formation processes they are interconnected, interdependent and inseparable in one hierarchical network structure. It seems that not a minor change can be made to any element of the structure.

- The formation processes start with different integers and as a result are capable to progress to different levels. This produces a complexity order in the formation processes and a sense that a formation process may have the aim to reach the level as higher as possible.

\section{Geometrization of the Integer Rela- tions}

In section 6 we discussed that in the formation processes the integer relations may be conveniently thought as some sort of "particles" transforming into each other. This intuitively pictures the formation processes more as a new resource similar to physical ones, rather than respects that they are formal constructs defined by the system of linear equations (6) and the inequality (7). However, integers and integer relations are known as abstract entities in the first place. They are not familiar as geometric objects suitable for observation and measurement.

Remarkably, it is possible to geometrize the integer relations as two-dimensional patterns and describe the formation processes of integer relations in terms of their geometric transformations [4]. This makes the theory of formation processes geometrical and quantitative. Furthermore, the geometrization visualizes the integer relations and their formations [4].

In particular, the areas of the geometric patterns can be used to describe quantitatively the formation process of integer relations and the strengths of the correlations in particular. As a result the integer relations produce a wide variety of 
numbers and suggest to consider whether their pattern may arise in experimental data.

We distinguish the PTM sequence, because it produces formation processes of integer relations progressing well through the levels of the hierarchy [4]. The area of a geometric pattern corresponding to an integer relation generated by the PTM sequence at level $\mathcal{N}$ of the hierarchy is given by $2^{\frac{\mathcal{N}(\mathcal{N}-1)}{2}} \varepsilon^{\mathcal{N}} \delta$, while the length - by $2^{\mathcal{N}} \varepsilon$. This allows us to control the area's growth by renormalization of length scales through levels. For instance, a level, where the area of the geometric pattern would equal its length, so that a renormalization of unit conditions $\delta=1$ and $\varepsilon=1$ may be possible, can be found from $2^{\frac{\mathcal{N}(\mathcal{N}-1)}{2}}=2^{\mathcal{N}}$. This gives $\mathcal{N}=3$.

\section{Conclusions}

In general, an irreducible theory that describes physical systems in terms of formation processes of integer relations is presented. It is suggested that properties of the formation processes may be used as irreducible guides in the search for a unified theory. Moreover, through the irreducibility of the formation processes it seems that a unified theory may look like:

- A unified theory may be based on a discrete hierarchical structure in the first place and not on a master equation formulated in space-time.

- The fundamental building blocks of a unified theory may exist. The other elements of the structure may be built from them by an organizing principle. This sets the relationships between the elements and makes them interconnected, interdependent and inseparable within one whole.

- The elements of an unified theory may be represented both arithmetically and geometrically.

- A unified theory may be formulated in geometrical terms as two-dimensional.
- The elements of a unified theory may provide nonlocal, instantaneous and inseparable correlations in space-time.

- A unified theory may have a complexity order and its properties may be interpreted with the purpose to progress in the direction of this order.

- The elements of a unified theory may be quantified and generate a pattern of numbers interrelated with each other.

\section{Acknowledgments}

I would like to thank Galina Korotkikh for useful discussions and comments. This work was supported by CQU Research Advancement Awards Scheme grant no. IN9022.

\section{References}

[1] S. Weinberg Dreams of a Final Theory (Pantheon, New York, 1992).

[2] M.B. Green, J.H. Schwarz and E. Witten, Superstring Theory (Cambridge University, Cambridge, 1987).

[3] L. Smolin, Three Roads to Quantum Gravity (Basic Books, 2001).

[4] V. Korotkikh, A Mathematical Structure for Emergent Computation (Kluwer, Dordrecht, 1999).

[5] G. Korotkikh and V. Korotkikh, in: Optimization and Industry: New Frontiers, ed. by P. Pardalos and V. Korotkikh (Kluwer, Dordrecht, 2003).

[6] V. Korotkikh, in: Fuzzy Partial Differential Equations and Relational Equations, ed. by M. Nikravesh, L. Zadeh and V. Korotkikh (Springer, Berlin, 2004).

[7] V. Korotkikh, Integer Code Series with Some Applications in Dynamical Systems and Complexity (Computing Centre of RAS, Moscow, 1993). 